\title{
Factors Impacting Animation in Icons for Visually Impaired Computer Users
}

\author{
Stephanie Ludi \\ Rochester Institute of Technology \\ Rochester, NY USA \\ salvse@rit.edu
}

\begin{abstract}
Icons are commonplace in Graphical User Interfaces. These same components that are intended to make computers easy to use can make technology difficult to access for the visually impaired. Eliminating icons would be unrealistic, but re-examining icons is a starting point in increasing accessibility in GUls. A project is underway where animation is being added to icons in order to assist visually impaired users who wish to access computing resources. Initially animated icons were not shown to be superior to static icons in terms of the size needed to identify them. This paper will present the analysis of some factors that influenced the results. The extent of the motion in the icons and the visual profile of the participants themselves contributed to the ability to identify animated icons. These insights will assist interface designers in developing more accessible Graphical User Interfaces.
\end{abstract}

Visually impaired, accessibility, graphical user interfaces, animation, icons

\section{INTRODUCTION}

Graphical User Interfaces are used to access a variety of resources in schools, the workplace, kiosks, and at home. A basic component of many Graphical User Interfaces are icons. Icons are the small static pictographs that represent a task that can be performed or a feature of the system. These icons, in conjunction with other aspects of Graphical User Interfaces, can make technology difficult to access for the visually impaired. Where magnification software is available the cost can be prohibitive, the software may not always be compatible or portable, and the user may find the constant scrolling to be frustrating. Also, people wish to utilize their remaining sight with minimum overhead. A response to the need to increase accessibility to computing devices that use GUls is to re-examine the interface components in order to enable the visually impaired users to utilize the same resources as those who have no visual impairment. The Animated Icon project focuses on re-inventing icons by adding animation to icons.

The overall objective of the initial study was to investigate how effective animation in icons can enable visually impaired computer users, those who possess functional vision, to utilize a Graphical User Interface in a similar manner as those who have no visual impairment. In the United States the legal definition of legally blind is when a person's vision is no better than $20 / 200$ in the better eye (measured with the Snellen vision chart) or posseses a visual field of 20 degrees or less [8]. For the study, a subset of visual acuity was selected for study, between $20 / 200$ and 20/800 (inclusive) with best correction. The notion of animated icons is not in itself innovative. Previous research has shown that the use of animation in icons can to increase the usefulness of icons in terms of clarification and ease of use [1, 2, 3] for mainstream users, but new applications of animation in the user interface may benefit the visually impaired population as well.

In order to fully gauge the scope of the accessibility issues in the computing industry, the work conducted in academia and industry was analyzed to assess the current state of affairs and to find gaps in current practice and the need of visually impaired workers. The results are to be used as the basis for future research and guidelines.

\subsection{Icon Design}

Icons are a basic component in Graphical User Interfaces. The small pictographs are used to represent applications, files, and functions within programs. A sample icon, representing the New Document function in Microsoft Word 98 is shown in Figure 1. Icons can be simple symbols or can contain pictures. The more concrete the symbol or picture, the stronger the association with the function that is being represented [4]. Icon design is critical in order to develop icons that will be effective in the parent software. Some general principles of effective icon design include:

- Icons in an interface should be unique

- Avoid icon confusion by eliminating the use of similar color schemes and appearance in close proximity

Accessible Design in the Digital World Conference 2005 
- Avoid orienting the pictograph that can confuse the purpose of the icon

- Use standard symbols when relevant [4]

When designed and laid out effectively, icons can allow users to access the functionality of software. Another attribute of icons is size, as their size tends to be small. For example, Microsoft Windows application icons are 24 $x 24$ pixels [5]. The use of icons in handheld devices and phones has further entrenched the use of small icons in interfaces.

FIGURE 1: New Document Icon in Microsoft Word 98

With the increased use of handheld devices and other technology in day-to-day life, icons need to be updated in order increase technology access by the visually impaired.

\subsection{Animation in Icons}

The use of animation in the user interface can serve several purposes as outlined in $[1,3,6]$. Animation can be used to provide an overview of menus, as is used in Microsoft Windows and the Macintosh OS. Animation can also be used to instruct the user in tutorials. Animation is also used to entertain and convey information. The use of animation as a means of demonstration is of importance in this study as animated icons can be used to "increase the amount of information that is contained in the iconic representation" and to "clarify the function of the icon through the use of animated rather than static symbols [6]. Especially novice or occasional users can also apply these benefits of animated icons as a means of aiding in the identification of the icons. The animation has added value over traditional static icons and has been shown to increase the comprehension of icons dramatically [3].

In use, animation must be controlled so as to not be distracting. User's can initiate the animation by placing the mouse over it and holding the pointer in the general area for a short period of time. Otherwise animated icons and animation in general, could be distracting to the viewer and detract from the overall interface. Thus far studies have been conducted with the general public, but the implication of animation as a means of improving access for the visually impaired is being undertaken here.

\section{RESEARCH QUESTIONS}

The overall project has a scope that falls beyond that of this paper. The initial study sought to find icon sizes that could later be used in the development of an interface using animated icons. The hypothesis was that if animated icons could be identified at a smaller size that traditional, static icons then the animated icons would be easier to identify. However after we learned that the absolute perception of animated icons at a smaller size than traditional static icons do not exist, new questions were presented. The study showed that some icons could be identified in a smaller state while animated [7]. The potential existed for the use of animated icons, but in order to better design animated icons further analysis is needed. What factors affected the participants' ability to identify an animated icon earlier than a static icon? These factors may be in the user population and/or in the icons themselves.

By examining these factors, animated icons can be designed so that their benefit would be more consistent and beneficial to the visually impaired users. Interface designers can use this information and apply it to interface designs and usability testing.

\section{METHOD}

\subsection{Participants.}

There were eight participants, who volunteered from the community. All participants possessed a visual acuity of $20 / 200$ or greater in each eye without correction. Five participants are legally blind. Due to the difficulty of locating participants who fit the criteria, three of the eight participants were used who fell into the criteria interval when not utilizing glasses or contact lenses. These three participants are referred to as possessing simulated legal blindness. No corrective aids were used during the study (e.g. magnifiers, glasses, contact lenses).

Besides the visual acuity data, other information regarding the participants is worthy of note. Of the eight participants, only three were male. The average age of the participants was 45.5 years old, where the youngest was 20 and the oldest was 79 years old. Also nearly all of the participants use computers at least 3 times per week. 


\subsection{Equipment.}

The experiments were conducted on PCs with 17-inch monitors, running Microsoft Windows 98. The screen size was $300 \mathrm{~mm}$ across and $225 \mathrm{~mm}$ tall and a resolution was maintained at 800 pixels by 600 pixels. As a result, each pixel had height and width dimensions of $.375 \mathrm{~mm}$. Due to the data collection methods used, the icon sizes are presented in pixels.

The icon identification program was developed using Macromedia Director 7. The program captured the size of each identified icon and saved it to a text file. The icons themselves were created using Macromedia Flash 4 . The icons and software development was conducted on a PC and a Macintosh G3.

\subsection{Design.}

The study used a 2-Forced Choice design for the test sets of animated icons and static icons. For each set, the participant selected the icon believed to match the task among two choices. The details will be presented in the Procedure section. The icons used for the study, shown in Figure 2, are representative of icons found in common application software including word-processors, email clients, and drawing programs.

Practice 1 (Selection)

Practice 2 (Underline)

Magnifier/Enlarge/Zoom

Pencil/Freehand Drawing

Right Arrow/Next
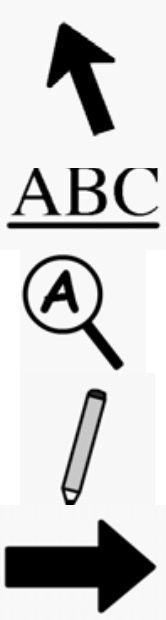

FIGURE 2: The ten icons used in the study.
Cut

Trash Can/Delete

Paste

Printer/Print

Floppy Disk/Save

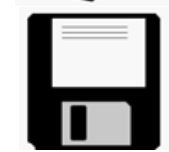

While the icons are meant to be commonplace, the icons were drawn rather than originating from pre-existing programs. A sampling of two animated icons is presented in Figure 3.

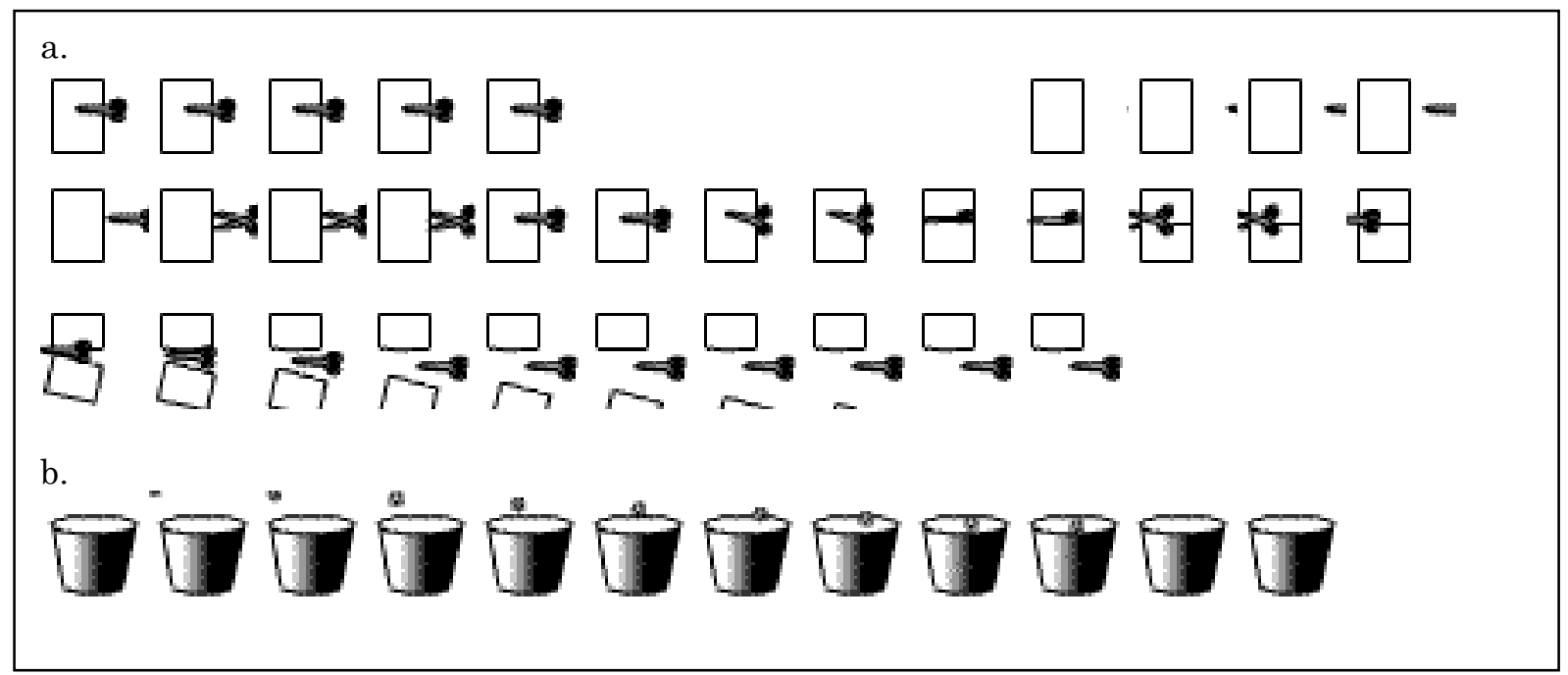

FIGURE 3: Sample of animated icons. a. Cut, b. Delete

All of the icons are in black-and-white, as the use of colour would not have been consistent and could have complicated the study. Animated icons were played at 9 frames per second, as that speed seemed to be a good 
balance between presenting motion fluidly though not too quickly so as for the purpose of the motion to be lost on the participant. Each animation varied in length, but most animations last approximately 4 seconds.

During the trials, abstract icons are used in order to provide noise for the participant. In order to see when the participant identified an icons, a similar icon needed to be a possible choice so that an accurate result can be obtained. Abstract icons, discussed in [9], were designed appear as a generalized version of each static and animated icon. In the case of animated icons, the movement of the icons was also generalized in appearance. The result was that the icons looked similar at the onset, but as the participant was able to identify the features and nuances of the icons, a valid selection could be made. The abstract version of each icon and the static icon it is associated with is presented in Figure 4.

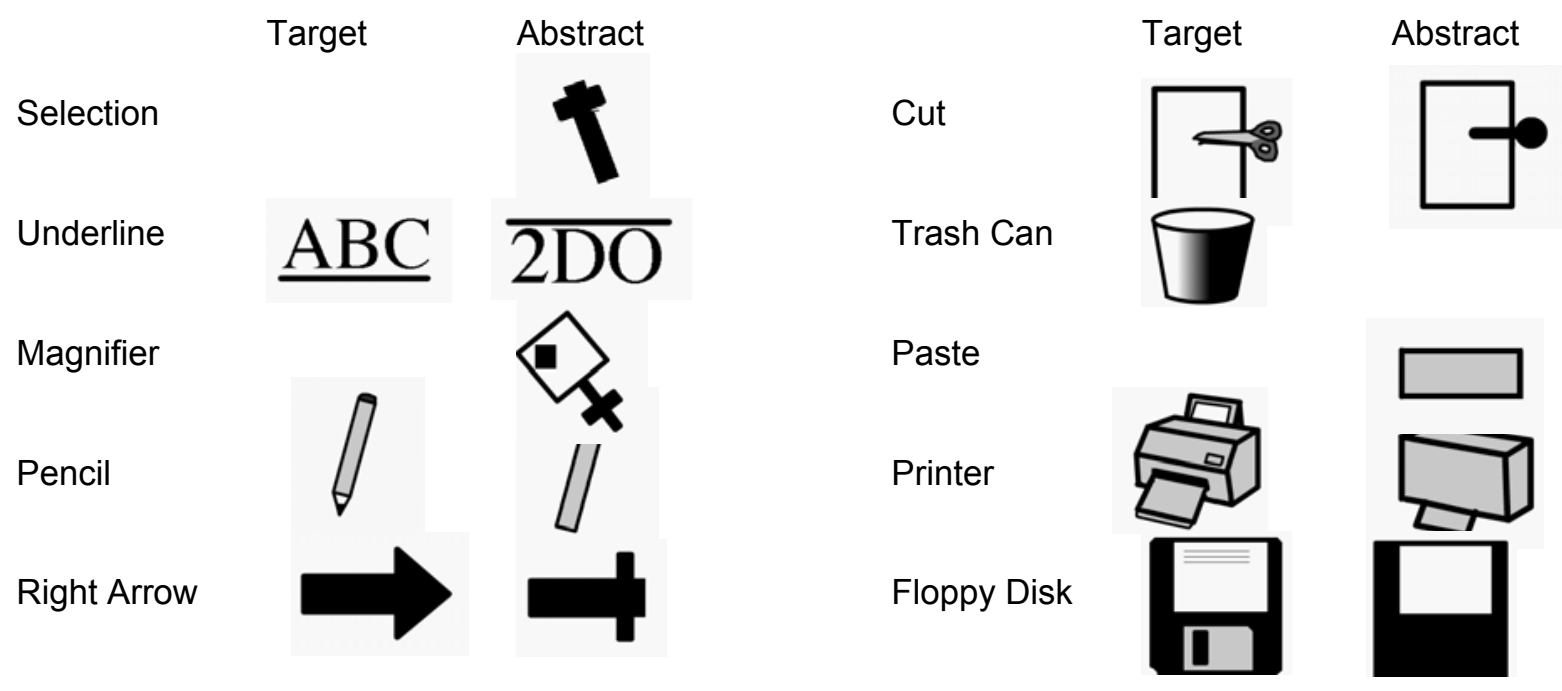

FIGURE 4: Abstract icons and the associated target icons

\section{PROCEDURE}

Before the first test set began, each participant answered several questions concerning general personal information (e.g. age), computer use, and visual condition. The trials were conducted with one participant at a time, sitting 19 inches away from the computer monitor.

The first set of trials was randomly chosen as either the animated icon set or the static icon set. The second trial was conducted a week later using the remaining icon set. For each trial within the set, one of the 10 icons was designated as the correct icon for a task while the abstract version of the correct icon was selected as noise. The first two trials in each set were practice icons, while the remaining 8 were used in the study. For each trial, the facilitator presented the icon's function to the participant. During each trial the "correct" icon and the "dummy" icon were each displayed for 10 seconds in a random order. The facilitator told the participant when the first icon was displayed and when the second icons were displayed. After both icons were displayed, the participant was required to select the number of the icon that was perceived as corresponding to the task. If the correct icon was selected, the program recorded the selection as a hit. The icon's width and height was then increased by 2 pixels until 3 consecutive hits occurred. Then the program recorded the icon size (from the first of the three consecutive hits) and proceeded to the next icon in the set.

\section{RESULTS}

The initial results were mixed, but held promise. As was presented in an earlier paper [7], animated and static icons were not identified at the same size. As shown in Table 1, static and animated icons were perceived at the same size in only 9 of the 66 trials (14\%). However no immediate trends appeared in terms of the size that the animated icons and static icons were identified. If animated icons could be identified at a smaller size, then the potential existed as a means to facilitate access by the visually impaired. However animated icons were perceived at a smaller size than the static version 33 times out of the 64 trials $(51.6 \%)$.

While the overall result does not offer overwhelming credibility calling for the use of animated icons, the examination of the data regarding specific icons shows that animation can make a difference. For example the Cut and Pencil icons show that the animated version was perceived at a smaller size than the static version in 7 out of the 8 trials for each icon. However other icons show that that particular animated version was not effective. For example the Delete version of the animated icon was identified at a smaller size than the static version in only 2 of 
the 8 trials. The results were promising and that other factors within the icons themselves and the participants contribute to the disparity in the data.

TABLE 1: Animated and static icon size comparisons shown as the number of trials. The Size Difference = static icon size animated icon size for each participant.

\begin{tabular}{lccc}
\hline Icon & Size difference $<0$ & Size difference $=0$ & Size difference $>0$ \\
\hline Arrow Right & 2 & 1 & 5 \\
Cut & 0 & 1 & 7 \\
Delete & 4 & 2 & 2 \\
Magnifier & 3 & 2 & 3 \\
Paste & 3 & 2 & 3 \\
Pencil & 1 & 0 & 7 \\
Print & 4 & 1 & 3 \\
Save & 5 & 0 & 3 \\
\hline Total trials & 22 & 9 & 33 \\
\hline
\end{tabular}

\section{DISCUSSION}

\subsection{Extent of Animation.}

The icons were animated in order to describe a task using motion. Since not all tasks use the same motion, the animations used in the icons also differ. Besides motion, the change in the amount of the image displayed on the monitor during the animation influences the exaggeration of motion and keeps the observers attention. The animated icons that were identifiable at a smaller size than the static versions were the Right Arrow, Cut and Pencil. In order to examine the motion in these and the other icons in order to ascertain any patterns, a graph was developed for each animated icon's motion. The generalized graphs present the amount of graphical material that is displayed over a period of time. The motion profiles for all eight icons are presented in Figure 5.

In order to examine patterns, the icons were divided into three groups. Group 1 contains the icons where the animated version was generally identified at a smaller size than the static version. The Right Arrow, Cut and Pencil icons are members of the first group. Group 2 consists of the icons where the static version was generally identified at a smaller size than the animated version. The Delete, Print and Save icons are in this second group. Lastly, group 3 consists of icons where no general identification pattern was apparent. The Magnifier and Paste icons in the third group show that an equal number of participants identified the animated icons at a smaller size than the static version as the number who identified the static icons at a smaller size than the animated version. The motion graphs for the icons in Group 1 are graphs A-C, Group 2 are graphs D-F, and Group 3 are graphs G and $\mathrm{H}$. 


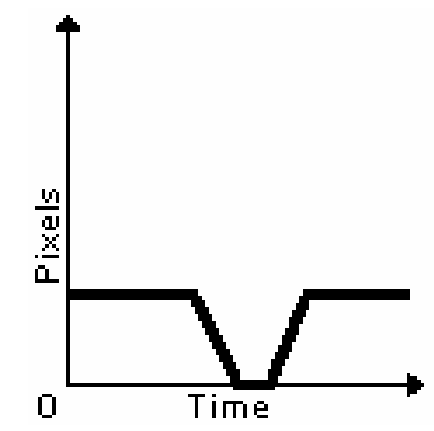

A. Right Arrow

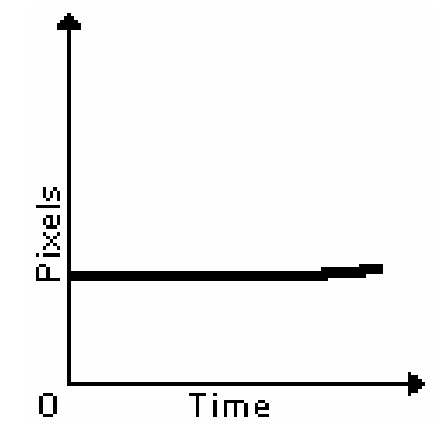

D. Print

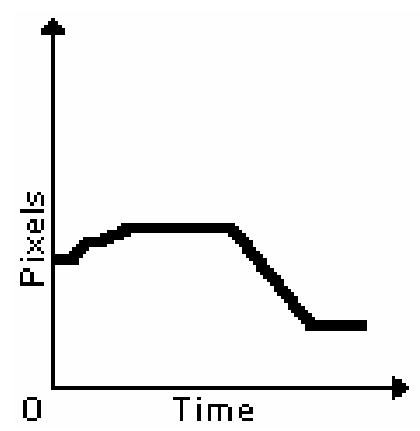

B. Cut

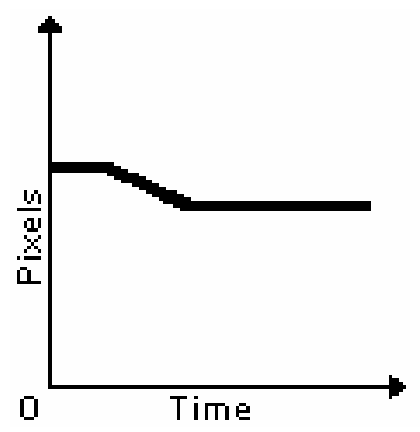

E. Save

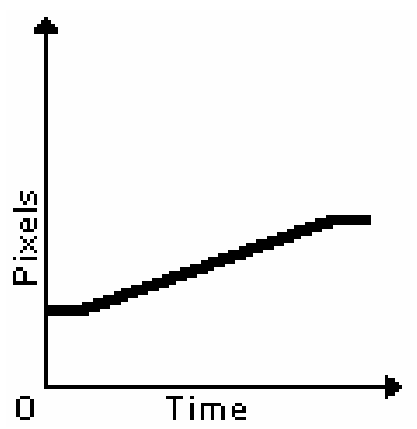

C. Pencil

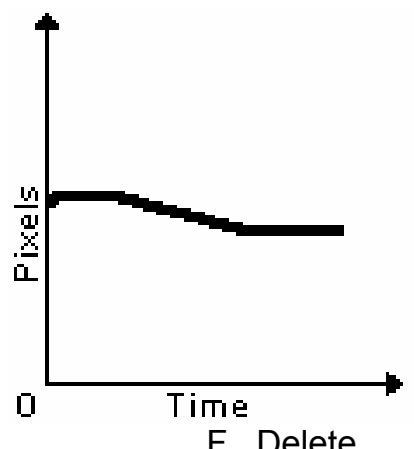

F. Delete

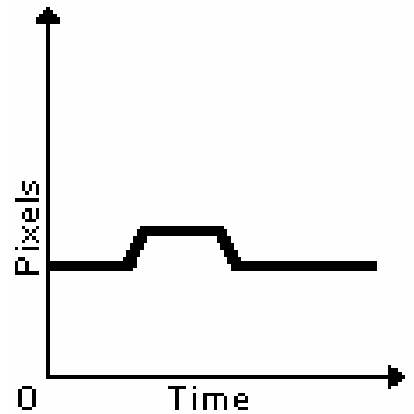

G. Magnify

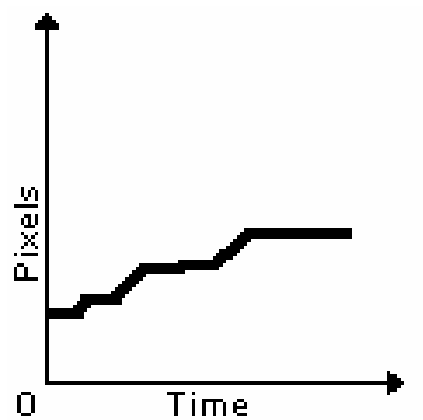

H. Paste

FIGURE 5: Representation of pixels displayed over time for each animated icon.

The common feature of the icons in Group 1 is that the motion is more dramatically exaggerated than the animation in the other groups. As the icons within this group were the icons that were identified at a small size when animated, the exaggerated movement allowed these animations attract and keep the viewer's attention. When the graphical difference is prominent the participant more likely to identify the icon earlier (and thus at a smaller size) than the static version.

By contrast the motion in the animated icons in Group 2 were more gradual, and in the case of the Print icon almost flat. This caused the participant to concentrate more on these icons and often become visibly frustrated at the lack of exaggerated motion in the animations.

The Magnifier and Paste icons in Group 3 were in the middle of the spectrum. As such these icons worked for some participants and did not work for an equal number of participants. The motions of these icons were not as exaggerated as those icons in Group 1, while not being gradual either. In the case of Paste, more and more material was added to the icon, but the steps were gradual and the impact of the motion was not absorbed to the extent as the Cut icon from Group 1.

The lesson learned in this icon analysis is that the motion in icons needs to be carefully designed. Exaggerated movements work best, especially since visually impaired users may not be able to perceive subtle or gradual changes. Once the animation in the icons is developed and tested, the icons can be viable interface components. Certainly these icons need to be enlarged in order to be identified as well and a range of options can be provided in order to be usable to a variety of users.

\subsection{Simulated Vision Loss.}

Although the study had a small sample size, the issue of simulated legal blindness versus legitimate legal blindness was apparent by the participant-level data. Of the eight participants, the visual acuity of three participants fell into the legally blind range without their glasses or contact lenses. With correction, the three 
participants' visual acuity was near or at 20/20. Since these participants functioned in the world with normal vision, their frame of reference would be different from the participants who functioned in the world with partial vision.

The main difference between the participants with simulated legal blindness and legitimate legal blindness lies in the size where the icons were perceived. The uncorrected visual acuity ranges of the simulated participants were between 20/200 and 20/400. Most of the visual acuity levels of the legitimately legally blind participants lay in the same acuity range. Even though the participants with correctable vision were asked to not wear their glass/contact lenses for several hours before the tests, the size differences were prominent. Many of the icons were identified at the same size at both the animated and static versions. When size differences occurred, the differences were no more than 12 pixels, with only minor exception. By contrast the size differences with the legitimately legally blind participants were often between 20 and 40 pixels. The differences in size are considerable.

While participants with simulated vision loss are used in usability testing, a cost exists. Recruiting normally sighted participants or participants with corrected vision are less expensive and easier then locating legally blind participants. Due to demographics or difficulties in travel, recruiting visually impaired participants can be difficult or not feasible in some geographical locations. However if you need the perspective of users who members of a particular user population, you should make a concerted effort to get input from these users. If our study was conducted using only participants with simulated legal blindness, the results would have been different and not as accurate.

\section{DESIGN IMPLICATIONS}

Improving the level of access to technology by all people is important as all of society benefits. The use of graphical elements, including icons, can be an obstacle for many visually impaired people. Incorporating animation into icons can address the needs of visually impaired individuals who possess some vision, but the animation must have exaggerated movements. When designing interfaces, details need to be examined. Even in the case of the animation in icons, not all animation is created equal. When motion is to be added into an icon, the icon designer needs to analyze the degree of motion occurring over time in order to create effective icons. One of the outcomes of the overall project will be large set of icons that can be used to guide developers.

In addition to the components themselves, the benefits of careful test planning are apparent. Recruiting legitimate representatives of your user population, as was the case of the legally blind population here, will enable you to get more accurate results then primarily using simulated representatives. Depending on your circumstances, partnering with a local low vision clinic or agency serving the visually impaired are options in recruiting subjects. The process can take time and you will need to be accommodating since travel can impact the ability to participate. The desire to participate in the effort to improve access to technology was clearly visible. So if you can spread the word and accommodate the unique needs of your subjects, you will get participants who will provide useful feedback for you interface or system.

When the visually impaired can gain better access to resources used in schools, the workplace, in public, and in the home their level of participation will increase. Examining current interfaces and updating them to meet the needs to the visually impaired can bring businesses new customers and bring schools new students. Animated icons are one answer among many that can re-invent the Graphical User Interface.

\section{ACKNOWLEDGEMENTS}

This study is part of a larger project funded by the National Science Foundation. (Award \#: IIS-9978183).

\section{REFERENCES}

[1] S. Alpert. (1991) Self-Describing Animated Icons for Human-Computers Interaction: a Research Note. Behaviour and Information Technology. 10, 2, (February 1991). 149-152.

[2] R. Baecker, I. Small and R. Mander. (1991) Bringing Icons to Life, Proceedings of CHI '91 Conference on Human Factors in Computing Systems, (April 1991). 1-6.

[3] J. Nielson. (2000) Designing Web Usability. New Riders Publishing, Indianapolis, 2000.

[4] A. Martin and D. Eastman. (1996) The User Interface Design Book. John Wiley and Sons, New York, 1996.

[5] Microsoft Windows Interface Components: Menus, Controls, and Toolbars (MSDN Online). http://msdn.microsoft.com/library/books/winguide/ch08.htm 
[6] R. Baecker and I. Small. (1990) Animation at the Interface in The Art of Human-Computer Interaction, edited by Brenda Laurel, Addison Wesley, Reading MA, 1990. 251-267.

[7] S. Ludi and M. Wagner. (2001) Re-inventing Icons: Using Animation as Cues in Icons for the Visually Impaired, Proceedings of the First International Conference on UAHCl, 2001. Publication pending.

[8] Social Security Administration Disability Benefits Document. http://www.ssa.gov/pubs/10052.html\#Blind

[9] D. Stots. (1998) The Usefulness of Icons on the Computer Interface: Effect of Graphical Abstraction and Functional Representation on Experienced and Novice Users, Proceedings of the Human Factors and Ergonomics Society 42nd Annual Meeting, (1998). 453-457. 